\title{
Sclerosing mesenteritis: update on diagnostic and therapeutic approach
}

\author{
César de Carvalho Garcia ${ }^{1}$, Senival Alves de Oliveira Júnior ${ }^{1}$, Aline Vasconcelos de Carvalho ${ }^{1}$, \\ Joanna de Andrade Cordeiro ${ }^{1}$, Bárbara Monitchelly Fernandes Chaves ${ }^{2}$, \\ Amália Cinthia Menezes Rêgo ${ }^{3}$, Irami Araújo-Filho ${ }^{4}$
}

\author{
${ }^{1}$ Medical Potiguar University, Laureate International Universities, Natal, Brazil \\ ${ }^{2}$ Federal University of Rio Grande do Norte, Natal, Brazil \\ ${ }^{3}$ Director of the School of Health Potiguar University, Laureate International Universities, Natal, Brazil \\ ${ }^{4}$ Department of General Surgery, Rio Grande do Norte Federal University, Natal, Brazil
}

Received: 28 February 2016

Accepted: 03 March 2016

\section{*Correspondence:}

Dr. Irami Araújo-Filho,

E-mail: irami.filho@uol.com.br

Copyright: (c) the author(s), publisher and licensee Medip Academy. This is an open-access article distributed under the terms of the Creative Commons Attribution Non-Commercial License, which permits unrestricted non-commercial use, distribution, and reproduction in any medium, provided the original work is properly cited.

\begin{abstract}
The sclerosing mesenteritis (SM) is a rare, non-specific inflammatory condition, mainly affecting the benign intestinal mesentery. It is known that she is diagnosed mainly during the sixth and seventh decade of life and seems to be two times more common in men than in women. The etiology of SM remains unknown, although several mechanisms have been suggested contributors, including surgery or abdominal trauma before, autoimmunity, paraneoplastic syndrome, ischemic injury and infections. The major signs and symptoms are abdominal pain, presence of palpable abdominal mass, nausea and vomiting, bowel changes, weight loss, small bowel obstruction, chylous ascites and peritoneal irritation signals. About of $10 \%$ of the patients are asymptomatic. The radiological study, especially computed tomography (CT) and magnetic resonance imaging (MRI) are essential components in the diagnostic evaluation. The "greasy" ring signal and the pseudocapsule tomographic findings are considered specific tumoral this pathology. The diagnosis is established by histopathologic study. There is no specific treatment for SM and should this be empirical and individualized. Although they are described cases of spontaneous remission, some authors have shown benefit with the empirical treatment using corticosteroids, colchicine, immunosuppressants, antibiotics, tamoxifen, alone or in combination. The surgical approach has a limited role and usually aimed at symptomatic relief. In most cases, the prognosis is favorable.
\end{abstract}

Keywords: Sclerosing mesenteritis, Mesenteric panniculitis, Retractile mesenteritis, Systemic nodular panniculitis, Liposclerotic mesenteritis, Mesentery

\section{INTRODUCTION}

The sclerosing mesenteritis (SE) is a rare condition, characterized by chronic inflammation and nonspecific of adipose tissue in the intestinal mesentery. ${ }^{1}$ Affects primarily the mesentery of the small intestine, and the involvement of the colonic mesentery observed in about $20 \%$ of cases. $^{2}$ May involve, even, more rarely, the fat, retroperitoneal and pelvic peripancreática. ${ }^{3}$ It was first described as mesenteritis retractable in 1924 and less than 300 cases were reported around the world. ${ }^{1}$
Presents various terminologies, such as mesenteritis, mesenteric panniculitis, nodular systemic panniculitis retractable, liposclerosis mesenteritis, mesenteric xanthogranulomatous, inflammatory pseudotumor and lipogranulomatosis. 2,3

Is diagnosed mainly during the sixth and seventh decade of life and seems to be two to three times more common in males than in females. Also, several reports indicate that to SM is more common in Caucasian men. Pediatric cases are exceptional, probably because children have 
less mesenteric fat, when compared with adults. ${ }^{1}$ Prevalence rates reported vary from $0.16 \%$ to $7.80 \%$. $^{4}$

The rarity of this condition restricts our study the demographic characteristics and clinical approach, as well as natural history and response to therapy disease. ${ }^{3-5}$ Thus, the therapeutic decisions are guided by anecdotal experience/empirical and case reports published in world literature. ${ }^{3}$ A more recent study described a prevalence of $0.6 \%$ in more than 7000 abdominal computed tomography examinations. ${ }^{5}$

The SM has unknown etiology and can occur independently or in association with other disorders. ${ }^{4}$ It is known that she represents the final stage of progression of chronic inflammatory diseases of the bowel mesentery, with predominance of fibrotic component. ${ }^{6}$

As a result, this clinical entity correlates with a number of pathologies, leading us to infer that it is a process nonspecific inflammatory, autoimmune, reactive to any kind of aggression, generating local inflammatory reaction intra-abdominal, whose diagnosis is based on the high clinical suspicion, histological and imaging studies, and these last two commonly required. ${ }^{2,5}$ The possible autoimmune etiology is supported by a significant clinical response to immunomodulatory drugs. ${ }^{2,5-7}$

Corticosteroids, immunosuppressants, colchicine, tamoxifen, progesterone, and recently the thalidomide, have been used with varying success. ${ }^{3}$ Despite the prognosis generally be favorable, about $20 \%$ of patients are associated with significant morbidity and a chronic course. ${ }^{8}$ The SM has a poorly understood association with underlying malignancy with contradictory results in the literature, which suggests it might be a paraneoplastic condition in at least some patients. ${ }^{4}$

The purpose of this article is to deepen the understanding of the main features of SM, with a focus on the pathogenesis, clinical presentation, diagnosis, differential diagnosis, treatment and prognosis, currently available on this entity.

\section{METHODS}

This work was done from an electronic search in the databases PubMed (Medline), Scopus, LILACS (Scielo) and Web of Science. We collected data from case reports, cohort studies and literary reviews, using the descriptors: Sclerosing mesenteritis; mesenteric panniculitis; retractile mesenteritis; systemic nodular panniculitis; liposclerotic mesenteritis; mesentery. The method presented the following guiding question: "What are the main results and scientific evidence identified in national and international bibliographical production of the last thirty years, pertaining to diagnostic and therapeutic approach of sclerosing mesenteritis?".
In the initial survey, 80 articles were identified, which have gone through evaluation of all researchers (authors), in accordance with the following inclusion criteria: articles published in Portuguese, French, English or Spanish, to submit the combinations of the keywords you selected, with a publication date from January 1986 to February 2016. After the initial selections of material were deleted the articles repeated in different databases and they focus on the other pathologies, the mixed sclerosing mesenteritis edge. Although picked by articles that cover effective updates in the treatment, the therapeutic failure was not used as a criterion for deletion, considering the particularity of the manifestations of each case. The final material featured 46 scientific articles.

\section{Pathogenesis}

To SM is described as an idiopathic inflammatory process followed by fibrosis that mainly affects the mesentery of the small intestine. According to recent literature, the involvement of the colonic mesentery is rare, as well as the peripancreatic region, omentum, retroperitoneum and pelvis. ${ }^{9,10}$

Although you do not have a defined cause, various mechanisms are being suggested based on case reports and animal studies. Among them, include abdominal trauma, previous surgery, autoimmune diseases, ischemic injury, and paraneoplastic syndromes, infections..$^{2-5,6,9,10}$

Emory reported a series in which $84 \%$ of patients had a history of trauma or any abdominal surgery. ${ }^{11}$ Durst et al stated that the recent surgery was related to $17 \%$ of its cases, constituting a predisposing factor. ${ }^{12}$ Abdominal surgeries more commonly described, cholecystectomy, appendectomy, hysterectomy and colectomy.,

Other factors, such as: gallstones, coronary heart disease, cirrhosis, abdominal aortic aneurysms, peptic ulcer or ascites, have also been related with this disease. ${ }^{13,14}$

Some authors consider that tobacco can also contribute to the onset of mesenteric panniculitis. As a result, the consumption of nicotine not only produces a sense of satisfaction that influences the neural reward system, but also has a wide variety of independent effects, such as an increased inflammatory response in the digestive tract. $^{13-15}$

The SM has been described in association with a number of conditions, including autoimmune thyroiditis, presumably of riedel, primary sclerosing cholangitis, retroperitoneal fibrosis, and orbital pseudotumor. In addition, can be related with paintings of autoimmune hemolytic anemia, minimum damage nephropathy, pulmonary fibrosis, lupus erythematosus and relapsing polychondritis. $^{2,5-7}$ Yet studies describe an intrinsic connection between the increase of serum IgG4 antibodies and the intestinal inflammatory process, 
suggesting a relationship between SM and IgG4-related diseases. 15

With regard to the paraneoplastic syndrome, the proportion of cases associated with underlying malignancy, varied in studies. Several neoplasms were reported, including lymphoma, breast cancer, melanoma, squamous carcinoma and pulmonary adenocarcinoma, renal carcinoma, multiple myeloma, chronic lymphocytic leukemia, mesothelioma thoracis, hepatocellular carcinoma, adenocarcinoma of the prostate, ovarian carcinoma, endometrial carcinoma, cervical carcinoma, angiosarcoma, gastrointestinal adenocarcinomas and carcinoid tumor.

The ischemic lesions are described correlated with the previous surgery, abdominal trauma, mesenteric thrombosis and mesenteric arteriopathy. ${ }^{3}$ The association with infections proved to be more evident in patients with personal history of infection with typhoid, dysentery, tuberculosis, syphilis, malaria, influenza and rheumatic fever. ${ }^{3-5,16}$ Initially a degenerative process of mesenteric fat, followed by inflammation (panniculitis). Later, develops and fibrosis the retractable mesenteritis appears. $^{9}$

The inflammatory process-fibrotic can affect the gastrointestinal lumen and the blood vessels and mesenteric lymph nodes, causing injury for mass effect, which may result in partial bowel obstruction, ischemia and ascites quilosa, justifying the diverse clinical gastrointestinal and systemic. ${ }^{3,10}$

Intraoperative findings might be the diffuse thickening of the mesentery, a single tumor, multiple tumors or also a various mix of the nodular and hypertrophic components. $^{17}$

Also the mesocolon may be involved, as well, rarely, mesoappendix, peripancreatic area, omentum and pelvis. Its gross appearance explains how easy it is to mimick many other intra-abdominal neoplastic diseases. Histology displays different bars of involvement. ${ }^{17-19}$

As regards the main complications, one can infer that they are intrinsically related to progressive fibrosis, shortening of the mesentery, intestinal obstruction (most often of small intestine) and ischemia by compression of blood vessels, which may require surgery. Ureteral obstruction is associated with retroperitoneal involvement. $^{18}$

\section{RESULTS}

The clinical presentation of SM is varied and its diagnosis requires a high degree of suspicion, because the

disease is usually asymptomatic. $5,19,20$
The major signs and symptoms appear to be associated with inflammation and the adjacent organ mass effect. $^{3-5,18}$

The duration of symptoms ranged from 24 hours to two years, with an average of 6 months, showing a continuous or intermittent character. ${ }^{5,18}$ About of $10 \%$ of the patients are asymptomatic at diagnosis, have being diagnosed when subjected to radiological procedures for other indications, including fever of undetermined origin or non-specific abdominal complaints. ${ }^{5}$

Other signs and symptoms may be present, and generally reflect conditions attached, such as an underlying malignancy. In these cases, the investigation the pleural effusion associated with mesothelioma, fever of unknown origin, protein-losing enteropathy, hemolytic anemia, jaundice by biliary obstruction and hardened subcutaneous nodules. ${ }^{5-7}$ The laboratory tests are often normal or discrete non-specific variations feature depending on the seriousness of the painting. There is anemia, neutrophilia, erythrocyte sedimentation rate (ESR) and levels of C-reactive protein (CRP), in addition to hypoalbuminemia. These changes are occasionally reported. The ESR and CRP can even serve as indirect markers of therapeutic response. ${ }^{10,18}$

The radiological study, particularly CT and MRI, are essential components in the diagnostic evaluation, though many of its features are nonspecific. ${ }^{5,6}$ The images vary according to the extent and the predominant component of the injury (inflammation, necrosis or fibrosis). ${ }^{19,20}$

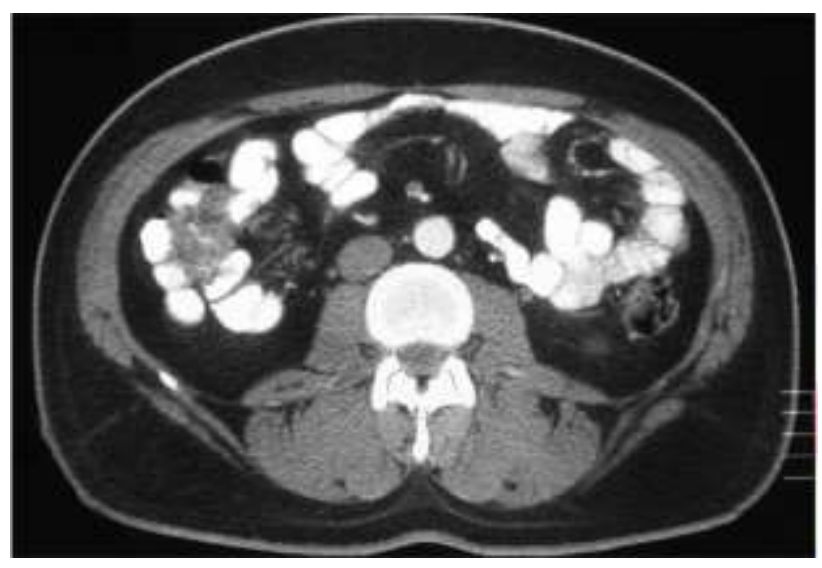

Figure 1: Axial contrast enhanced CT image shows displacement of small bowel loops by the well-defined fatty mass in the mesentery. Notice that the mesenteric vessels are not displaced but rather engulfed in the mass.

The abdominal CT (Figure 1), is the most sensitive imaging method to evaluate the presence of the disease, however, their specificity is limited in function of the wide differential diagnosis of mass lesions of the mesentery. ${ }^{5,21}$ The most common finding is a nonspecific mass of soft tissue in the mesentery of the small intestine, 
which infiltrate the intestinal tissue..$^{7-10,18}$ If the image presents a homogeneous texture, we can think of a lesion with predominantly fibrotic, and component when heterogeneous, suggests the predominance. The mesentery of jejunum seems to be the most commonly involved, whereas in the sigmoid colon involvement is as described in the studies. $5,9,18-21$

The most striking feature of the TC, SM is the increase in the density of mesenteric fat for attenuation values of -40 to -60 Hounsfield units (HU), compared with the normal attenuation of subcutaneous fat and retroperitoneum, which are, respectively, -100 to $-160 \mathrm{HU} .^{2,3}$

The "greasy" ring signal (75-90\% of cases) and the tumor pseudocapsule (50-60\% of cases), are considered specific findings of this pathology. The sign of the greasy ring represents the soft tissue surrounding the perivascular fat without infiltrate the mesenteric vessels, while the pseudocapsule tumor refers to the presence of a hiperatenuante band that surrounds the mass lesion. These findings are important not only for diagnosis, but also to the exclusion of other tumors, like the lipomatosos, especially the liposarcoma lipogenic, carcinoid tumor and lymphoma., $3,18,21$

Calcifications are present in about $20 \%$ of the tomographic findings and probably result from fat necrosis. ${ }^{18-22}$ The retroperitoneal or mesenteric lymphadenomegaly, is present in $20-40 \%$ of patients, and generally displays nodules smaller than $5 \mathrm{~mm}$ in diameter. $^{5-8}$

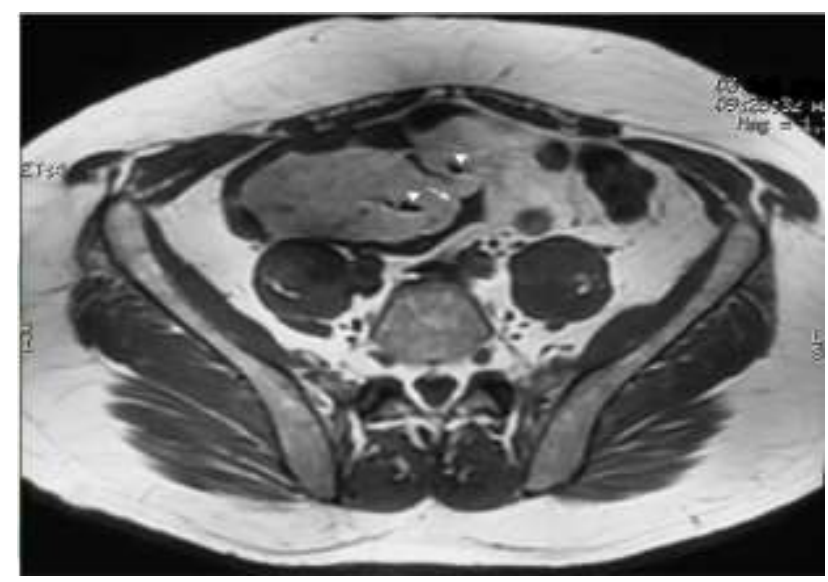

Figure 2: Axial T1-weighted MR image showing the mass with intermediate-low signal intensity in the mesentery that displaces small bowel loops which have a speculated and irregular outline (arrow). Flow void correspond to lumen of mesenteric vessels that are engulfed in the mass but not displaced (arrowheads).

The role of the TC is not limited only to the diagnosis of this disease, but also to non-invasive monitoring of the progression of volume and mass, as well as extension of the vascular involvement and identification of potential complications. In addition, AIDS in exclusion of diseases requiring urgent surgical intervention.,

The MRI (Figure 2) has an important role in the diagnosis of SM, since it allows for better characterization of tissue. In T1-weighted sequences, the lesion presents intermediate signal, while in the T-2, the standard signal may vary according to the predominant tissue, that is, if there are severe fibrosis, there will be hypointense signal, while if there is a predominance of inflammatory process and residual fat, there will be hiperssinal. ${ }^{18}$

The ultrasound appearance of SM is variable and nonspecific, showing both descriptions with heterogeneous masses (predominantly hyperechoic) as hypoechoic masses. The ultrasound with color doppler, can clearly show the mesenteric vessels within the masses. $^{18}$

Computed tomography positron emission tomography (PET) and angiography, have also been used to detect this condition, but the literature is still scarce. ${ }^{9}$ The PET was proposed as a promising tool in differentiation between benign and SM with lymphoma tumor involvement mesenteric. ${ }^{3}$ Because of this, the diagnosis is established by histopathologic study, being the essential biopsy and mandatory on all suspected cases. So, despite the percutaneous biopsy can be used to obtain the tissue, laparoscopy or laparotomy, are often required. ${ }^{5,22}$

Histologically, the disease progresses in three stages. The first phase is characterized by mesenteric lipodystrophy, in which a layer of foamy macrophages replaces mesenteric fat. Right now, the acute inflammatory signs are minimal or non-existent, and the disease tends to be clinically asymptomatic with a good prognosis. ${ }^{9,10}$

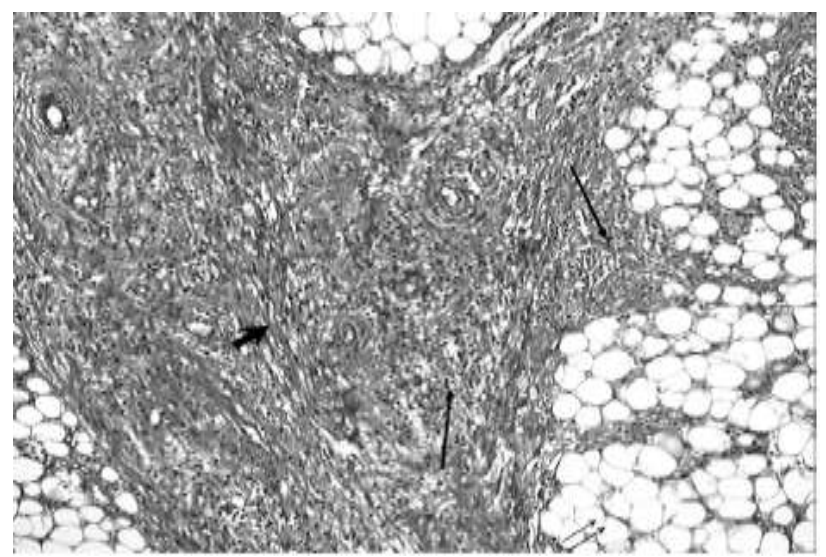

Figure 3: Fibroadipose tissue with areas of adiponecrosis (double arrows), foci of mononuclear cell from infiltrate inflammatory (arrows) and proliferation of bundle-forming fibroblasts (large arrow) (Hematoxylin-eosin, $\times 100$ ).

In the second step (Figure 3), called mesenteric panniculitis, histology reveals an undercover consisting 
of B cells, polymorphonuclear leukocytes and macrophages sparkling. ${ }^{9,10}$ The segments of the small intestine may show slight thickening and elongation. The most common symptoms include fever, abdominal pain and malaise. $^{23}$

Already in the final stage, adopts the sclerosing term, that has a stage with deposition of collagen, fibrosis, inflammation, and, occasionally, calcifications. The deposition of collagen leads to scarring and shrinkage of the mesentery. ${ }^{9,10}$ This leads to the formation of an abdominal mass, leading the obstructive symptoms. ${ }^{24}$

Some studies suggest diagnostic criteria based on radiological and histopathological findings: a) injuries with fat density that configure single, multiple or diffuse masses; b) histological confirmation of fat necrosis with inflammatory infiltrate and/or infiltration by macrophages; c) no evidence of inflammatory bowel disease, pancreatitis or extra-abdominal fat necrosis (suggestive of Weber-Christian disease). However, these criteria require more studies still to be properly established. ${ }^{5,18}$

\section{Differential diagnostics}

The differential diagnosis of SM is broad and includes all diseases that can affect the mesentery. Among them can be mentioned: Lymphoma, peritoneal carcinomatosis, carcinoid tumor, desmoid tumor, peritoneal mesothelioma, amyloidosis, chronic inflammation due to foreign body, metastasized from desmoplastic carcinoma, sarcoma, retroperitoneal, mesenteric fibromatosis and mesenteric edema. Mesenteric edema can be caused by a number of conditions, including hypoalbuminemia, cirrhosis, congestive heart failure and traumas. ${ }^{5,9,25}$

Other diagnoses should be considered. These include primary inflammatory diseases of the pancreas (acute pancreatitis) and intestine (inflammatory bowel disease), systemic diseases with fat necrosis (Weber-Christian disease), peritoneal inflammatory pseudotumor, lymphomatoses, idiopathic retroperitoneal fibrosis, lipoma, liposarcoma, deposits, tumor infiltration by adenocarcinoma of the gastrointestinal tract, sclerosing peritonitis, Whipple's disease, mesenteric cysts, and peritoneal mycobacterial diseases. 5,6

\section{DISCUSSION}

There is no consensus of opinion on medical treatment for symptomatic cases of SM and many clinicians develop a therapeutic approach based on the patient's clinical scenario, with a frequent focus on the aspects of the inflammatory disease process. $^{26,27-31}$ Some investigators report spontaneous resolution of disease, and others describe the curative resection and laparotomy is clearly indicated in the case of sclerosing mesenteritis, associated bowel obstruction nonresolving. ${ }^{2,29-33}$
In the absence of spontaneous resolution, several different therapeutic approaches have found some measure of success; although in the therapies are uniformly efficacious. ${ }^{34}$

The clinical management depends on the histological findings and stage of the disease. In the early stages, when fat necrosis is the main feature, it tends to settle spontaneously without. ${ }^{26,35}$ As the disease progresses and chronic inflammation with or without fibrosis predominates, various agents have been used alone or in combination. Treatment has been attempted with a variety of drugs including steroids, azathioprine, cyclophosphamide, colchicine, tamoxifen, or radiotherapy, with different degrees of success. ${ }^{36-38}$

The patients with a greater inflammatory component, with fever, weight loss, and general malaise appear to be the most receptive to glucocorticoids, alone or in combination. $^{39}$

Some cases have been related reports successful role of colchicine therapy in the maintenance of remission of mesenteric panniculitis. ${ }^{10,38}$ Mechanisms by which colchicine acts under pathological conditions are speculative and include: (a) binding $\beta$-tubulin and $\beta$ tubulin-colchicine making pachycondyla, which inhibit the assembly of microtubules and the mitotic spindle formation; (b) modulation of the production of chemokines and prostanoids; and (c) inhibition of neutrophils and endothelial cell adhesion molecules. ${ }^{40}$

Pentoxyfylline has been recently reported the promising antifibrotic agent successfully used in a case of sclerosing mesenteritis. ${ }^{3}$ Tamoxifen was successfully used in 19 patients in the series of Akram et al and $63 \%$ of these patients responded within 12 weeks of initiation of treatment. ${ }^{41}$ In the same series in similar benefits were obtained with prednisone alone or in combination with non-tamoxifen treatment. The intense fibrosis bowel obstruction may occur appears. Bowel resections, bypasses or ostomy might be required. ${ }^{41}$

Performing surgical resection of the lesion is quite difficult due to the presence of associated vascular compromise and the extent of disease. ${ }^{42}$ The surgical approach should be limited to biopsy of the mass. ${ }^{43}$ Therefore, surgery should be attempted only in patients with severe complications, such as bowel obstruction or perforation and advanced inflammatory changes irreversible. $^{42}$

Thus, researches related surgical resection must be individualized according to patient's anatomic characteristics due to the possibility of complications arise, becoming the worst life quality. For example, if a long segment of the jejunum would have needed to be removed in the patient with small bowel mesentery of the jejunum which was shown to be widely involved on the CT scan and gross findings during laparotomy, such an 
operation could, with high probability, result in short bowel syndrome. ${ }^{44}$ Although there is uncertainty about the optimal treatment, it is a situation with a favorable prognosis in most patients with good responses to treatment. $^{6}$

\section{Prognosis}

The natural history of sclerosing mesenteritis is not well understood. This happens due to the rarity of this condition, as well as the confused nomenclature which disperses in epidemiological reports and the lack of proper monitoring. ${ }^{5}$

The sclerosing mesenteritis has usually a benign, selflimited course, slowly progressive evolution and resolves spontaneously in most cases, showing a favorable prognosis. This mainly depends on a correct diagnosis and extent of fibrotic process. The rate of recurrence is rare. However, $20 \%$ of patients have significant morbidity and a chronic debilitating course..$^{5,6,45}$

Because of this, the more advanced stages seem to attend with a negative and less therapeutic response. A minority of patients have rapid and fatal evolution, and according to some authors, a reasonable percentage developed malignancies during the studies, including lymphoma, carcinoid syndrome, lung adenocarcinoma and mesothelioma. ${ }^{6,45}$

In the series of Daskalogiannaki et al the sclerosing mesenteritis was related to malignancy in $69 \%$ of the patients. The association with malignancy can be coincidental or secondary to autoimmune inflammatory reaction. However, the exact mechanism has yet to be elucidated. ${ }^{46}$ It is known that the prevalence of previous and/or concomitant malignancy, was significantly higher in patients with SM than in the control group. This was particularly true for the prevalence of prostatic carcinoma. ${ }^{4}$

The duration between the initial diagnosis of SM and the diagnosis of malignancy, ranged from 2 months to 4 years and 4 months, with an average of 19 months. However, not found signs of malignancy lows CT exams, for patients who developed after the diagnosis of malignancy $\mathrm{SM}^{4}$

In another study involving 10 patients, three showed a history of gastrointestinal tumors (pancreas, stomach and colon), with a time span of 7, 10 and 36 months, respectively, after the diagnosis of mesenteric panniculitis, and underwent pancreaticoduodenectomy, total gastrectomy and sigmoid, respectively. ${ }^{1}$

Due to the scarcity of information and the importance of the association between mesenterite and sclerosing malignancies, it is essential that more study and research be carried out to elucidate such an association.

\section{CONCLUSION}

In summary, one can infer that mesenterite is a rare, sclerosing and constitutes a challenge for many professionals, the example of the surgeon, radiologist, pathologist and gastroenterologist. In addition, it is essential that this pathology is always included in the differential diagnosis of patients with severe systemic manifestations and high acute inflammatory response markers of unknown etiology, especially when there are demonstrations in topography of abdomen.

Funding: No funding sources

Conflict of interest: None declared

Ethical approval: Not required

\section{REFERENCES}

1. Delgado L, Rodríguez E. Mesenteric panniculitis: Experience in our center. Rev Esp Enferm Dig. 2007;99:291-7.

2. Galvão-alves J, Galvão MC, Cavalcante D. Relato de caso - Mesenterite. J. bras. Med. 2013;101:54-6.

3. Vlachos K, Archontovasilis F, Falidas F. Sclerosing Mesenteritis: Diverse clinical presentations and dissimilar treatment options. A case series and review of the literature. Int Arch Med. 2011;17:1-9.

4. Van Putte-Katier N, Van Bommel EFH, Elgersma OE, Hendriksz TR. Mesenteric panniculitis: prevalence, clinicoradiological presentation and 5year follow-up. $\mathrm{Br} \quad \mathrm{J}$ Radiol. 2014;87(1044):20140451.

5. Masulovic D, Jovanovic M, Ivanovic A, Stojakov D, Micev M. Sclerosing Mesenteritis Presenting as a Pseudotumor of the Greater Omentum. Med Princ Pract. 2016;25:93-5.

6. Ferreira M, Silva A, Meruje M. Mesenterite Esclerosante. Acta Med Port. 2009;22:855-60.

7. Gundara JS, Ip JC, Glover AR. Sclerosing mesenteritis: a diagnosis worth considering. ANZ Journal of Surgery. 2015;85:291-2.

8. Cormack OM, Meaney JF, Reynolds JV. Resolving sclerosing mesenteritis. Surgery. 2012;153:879-80.

9. Ramachandran V. An Unusual Case of Intestinal Obstruction, Tropical Gastroenterology. 2009;30:222-5.

10. Fasoulas K, Beltsis A, Katsinelos T. Efficacy of Colchicine in the Treatment of Mesenteric Panniculitis in a Young Patient. The Saudi Journal of Gastroenterology. 2012;18:146-8.

11. Emory TS, Monihan JM, Car NJ, Sobin LH. Sclerosing mesenteritis, mesenteric panniculitis and mesenteric lipodystrophy: a single entity? Am J Surg Pathol. 1997;21:392-8.

12. Durst AL, Freund H, Rosenmann E, Birnbaum D. Mesenteric panniculitis: Review of the literature and presentation of cases. Surgery. 1977;81:203-11.

13. Patel N, Saleeb SF, Teplick SK. Cases of the day Mesenteric panniculitis with extensive inflammatory 
involvement of the peritoneum and intraperitoneal structures. Radiographics. 1999;19:1083-5.

14. Janisch HD, von Kleist D, Pfretzschner D, Szekessy T, Pfannkuch F. Mesenteric lipodystrophy: differential diagnosis of a benign abdominal tumor. Wien Med Wochenschr. 1984;134:417-9.

15. Kim EJ, Kim EY, Song JE. A Case of IgG4-Related Sclerosing Mesenteritis Associated with Crohn's Disease. Korean J Gastroenterol. 2014;63:176-82.

16. Guettrot-imbert G, Boyer L, Piette JC. Panniculite mésentérique. La Revue de médecine interne. 2012;33:621-7.

17. Montgomery E, Torbenson MS, Kaushal M, Fisher C, Abraham SC. Beta-catenin immunohistochemistry separates mesenteric fibromatosis from gastrointestinal stromal tumor and sclerosing mesenteritis. Am J Surg Pathol. 2002;26:1296-301.

18. Moreira LB, Pinheiro RA, Melo AS. Paniculite Mesentérica: Aspectos na Tomografia Computadorizada. Radiol Bras. 2001;34:135-40.

19. Mitchell A, Caty V, Bendavid Y. Massive mesenteric panniculitis due to fibromuscular dysplasia of the inferior mesenteric artery: a case report. BMC Gastroenterol. 2015;23;15:71.

20. Seo M, Okada M, Okina S, Ohdera K, Nakashima R. Mesenteric panniculitis of the colon with obstruction of the inferior mesenteric vein: report of a case. Dis colon Rectum. 2001;44:885-9.

21. Preda M, Buchan C, Preda T. Sclerosing mesenteritis presenting with abdominal pain and fever in a young woman: computed tomography findings. ANZ J Surg. 2008;78:709-10.

22. Parra-Buitrago A, Valencia-Zuluaga N, RiveraEcheverry J. Idiopathic sclerosing mesenteritis in the pediatric patient: A case report. Revista de Gastroenterología de México. 2013;78:255-60.

23. Kelly JK, Hwang WS. Idiopathic retractile (sclerosing) mesenteritis and its differential diagnosis. Am J Surg Pathol. 1989;13:513-21.

24. Bashir MS, Abbott CR. Mesenteric lipodystrophy. J Clin Pathol. 1993;46:872-4.

25. Plasencia LD, Ballester LR, López-Tomassetti EMF, Morales AH, Pallarés AC. Mesenteric panniculitis: Experience in our center. Rev esp enferm dig. 2007;99:291-7.

26. Daumas A, Agostini S, Villeret J, Ah-Soune P, Emungania O. Spontaneous resolution of severe, symptomatic mesocolic panniculitis: a case report. BMC Gastroenterology. 2012;12:59-60.

27. Grieser C, Denecke T, Langrehr J. Sclerosing Mesenteritis as a Rare Cause of Upper Abdominal Pain and Digestive Disorders. Acta Radiol. 2008:13.

28. Kapsoritakis AN, Rizos CD, Delikoukos S. Retractile mesenteritis presenting with malabsorption syndrome. Successful treatment with oral pentoxifylline. J Gastrointestin Liver Dis. 2008;17:91-4.
29. Lim CS, Singh RG, Tibrewal S. Sclerosing mesenteritis presenting with small bowel obstruction and subsequent retroperitoneal fibrosis. Eur J Gastroenterol Hepatol. 2006;18:1285-7.

30. Mazure R, Fernandez MP, Niveloni S. Successful treatment of retractile mesenteritis with oral progesterone. Gastroenterology. 1998;114:1313-7.

31. Rader M, Cofer J. Sclerosing mesenteritis masquerading as a small bowel mesentery neoplasm. Am Surg. 2008;74:743-5.

32. Ondrejka P, Faller J, Sapi Z. Mesenteric panniculitis spreading to the pancreas simulating pancreatic neoplasm. Endoscopy. 1998;30:S94-5.

33. Sheikh RA, Prindiville TP, Arenson D. Sclerosing mesenteritis seen clinically as pancreatic pseudotumor: two cases and a review. Pancreas. 1999;18:316-21.

34. Sabate JM, Torrubia S, Maideu J. Sclerosing mesenteritis: imaging findings in 17 patients. AJR Am J Roentgenol. 1999;172:625-9.

35. Issa I, Baydoun H. Mesenteric panniculitis: Various presentations and treatment regimens. World $\mathbf{J}$ Gastroenterol. 2009;15:3827-30.

36. Kikiros CS, Edis AJ. Mesenteric panniculitis resulting in bowel obstruction: Response to steroids. Aust N Z J Surg. 1989;59:287-90.

37. Bush RW, Hammar SP, Rudolph RH. Sclerosing mesenteritis. Response to cyclophosphamide. Arch Intern Med. 1986;146:503-5.

38. Iwanicki-Caron I, Savoye G, Legros JR, SavoyeCollet C, Herve S. Successful management of symptoms of steroid-dependent mesenteric panniculitis with colchicine. Dig Dis Sci. 2006;51:1245-9.

39. Parra-Buitrago A, Valencia-Zuluaga NA, RiveraEcheverry JA, Contreras-Ramírez M, Vélez-Hoyos A. Idiopathic sclerosing mesenteritis in the pediatric patient: A case report. Rev gastroenterol mex. 2013;78:255-7.

40. Cerquaglia C, Diaco M, Nucera G, La Regina M, Montalto M. Pharmacological and clinical basis of treatment of familial Mediterranean fever with colchicine or analogues: An update. Curr Drug Targets Inflamm Allergy. 2005;4:117-24.

41. Akram S, Pardi DS, Schaffner JA, Smyrk TC. Sclerosing mesenteritis: clinical features, treatment, and outcome in ninety-two patients. Clin Gastroenterol Hepatol. 2007;5:589-96.

42. Parra-Davila E, McKenney MG, Sleeman D. Mesenteric panniculitis: case report and literature review. Am Surg. 1998;64:768-71.

43. Daumas A, Agostini S, Villeret J, Ah-Soune P, Emungania O. Spontaneous resolution of severe, symptomatic mesocolic panniculitis: a case report. BMC Gastroenterol. 2012;12:59.

44. Endo KK, Moroi R, Sugimura M, Fujishima F, Naitoh T. Refractory sclerosing mesenteritis Involving the Small Intestinal Mesentery: A Case Report and Literature Review. Intern Med. 2014;53:1419-27. 
45. Rispo A, Sica M, Bucci L. Protein-loosing enteropathy in sclerosing mesenteritis. Eur Rev Med Pharmacol Sci. 2015;19:477-80.

46. Sumita C, Satheesh Y. An unusual presentation of sclerosing mesenteritis as pneumoperitoneum: case report with a review of the literature. World $\mathbf{J}$ Gastroenterol. 2009;15:117-20.
Cite this article as: Garcia CC, Júnior SAO, Carvalho AV, Cordeiro JA, Chaves BMF, Rêgo ACM, et al. Sclerosing mesenteritis: update on diagnostic and therapeutic approach. Int Surg J 2016;3:456-63. 